Article

\title{
Impact of Crude Methanolic Extract of Melia azedarach L. on The Fecundity and The Cuticle of The Adult House Fly, Musca domestica Macq
}

\author{
Radwan, E.H. ${ }^{1,2}$, Youssef, N.S. ${ }^{2}$, Hashem, H.O. ${ }^{2}$ and Shalaby A.M. ${ }^{2}$ \\ 1. Damanhour University, Faculty of science, Zoology Department, Egypt. \\ 2. Alexandria University, Faculty of Science, Zoology Department, Egypt \\ 3. Member of National Biotechnology Network of Expertise, ASRT, Egypt \\ Corresponding author dr_eman_hashem@yahoo.com, eman.radwan@sci.dmu.edu.eg
}

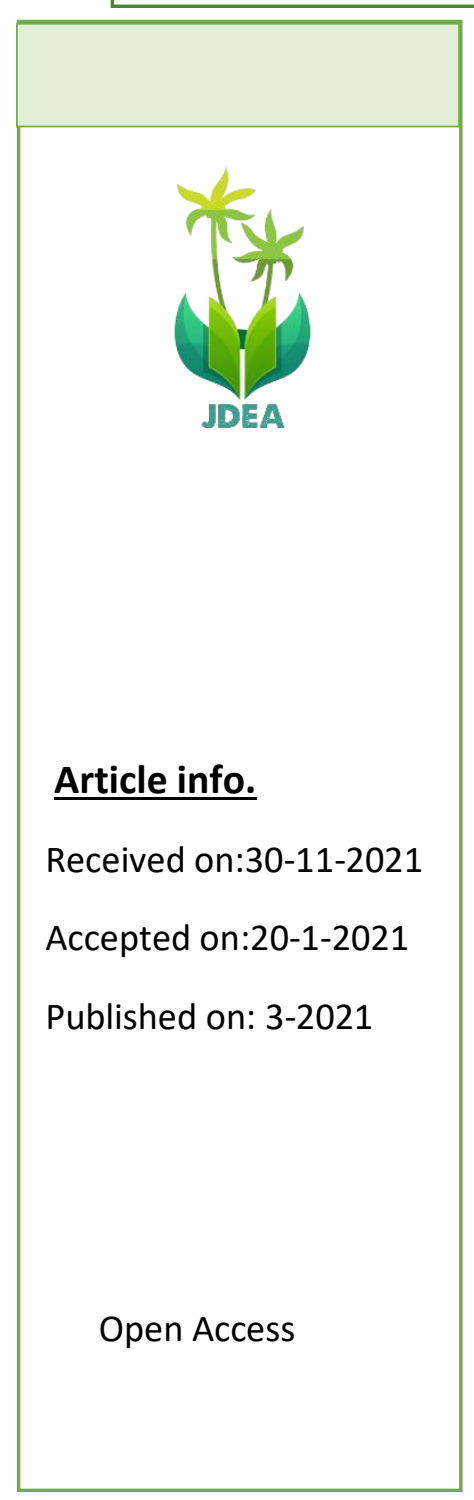

\section{Abstract}

The house fly Musca vicina Macq is found in human habitat. Insecticides usually destroy the natural enemies of a given pest more than the pest itself. The penetration of substances, through the integument of a living insect, is restricted by the structure of the cuticle. Numerous studies have been carried out on the insecticidal properties of different plant extracts. Family Meliaceae represents one of the most important plants in this respect, as it contains the neem tree Azadirachta indica A. Juss and the chinaberry tree Melia azedarach (the Egyptian common name is Zanzalacht). The discovery of insecticidal properties of Melia azedarach created quite a stir among entomologists interested in the practical uses of insect.

The Musca domestica vicina, dissected and examined in this study, were all produced from a colony raised at the laboratory of the Department Zoology, Faculty of Science, University of Alexandria. Fresh leaves and ripe fruits were collected from the chinaberry trees, Melia azedarach, found in the garden of Faculty of Science at Shatby, Alexandria University. Leaves and fruits were washed in running tap water and air dried for several days, then were put in an oven at $60^{\circ} \mathrm{C}$ until reaching a constant weight, afterwards, they were pulverized by using a hummer mill. Extraction was conducted in a $250 \mathrm{ml}$ Soxhlet apparatus using methanol as a solvent. However, no separation of fat materials was done. The extraction period lasted a total of 20 hours over four days until the chinaberry leaves and fruits became colorless. The fly fertility was determined as the number of eggs hatched in relation to the total number of eggs laid. Results showed that adults fertility was affected by treatment with Melia azedarach extract.

$\underline{\text { Key words: }}$ house fly-natural insecticides-topical application-fertility. 


\section{Introduction}

The house fly Musca vicina Macq is present in human habitations. As a vector of some serious diseases like typhoid, and colera, this species has become common in crowded cities. Housing developments accentuate the fly problem because they provide new and sometimes better habitats for this insect. Modern fly control tactics involve three approaches, namely source reduction; use of biological control techniques and control by insecticides (El Ahwany et al., 2016). Insecticides play an important role in the overall fly population suppression programs. insecticides usually have adverse impact on populations of the natural enemy complex of a given pest insect hormones were also utilized and their impact was assessed. Topical application of Melia azedarach extract of the newly emerged female flies causes the production of abnormal forms that fail to emerge normally and soon die (Radwan et al., 2019a, Ochi et al., 2020).

Employing Melia azedarach may give similar results as using chemical insecticides, but with different characteristics and modes of action. Unlike conventional insecticides, Melia azedarach compounds are not toxicant, instead they disrupt normal physiological processes associated with development and metamorphosis. Recent investigations revealed that the conventional insecticides are toxic to humans and beneficial natural enemies (Eissa et al., $2020 \mathrm{a}, \mathrm{b}$ ). There has been renewed interest in the development of repellents and feeding deterrents as alternatives to insecticides to avoid their adverse impact on the environment (Radwan et al., 2019 a, b, c ).

The penetration of substances, through the integument of a living insect, depends on the structure of the cuticle (Ochi et al., 2020). It is for this reason that the study of the adult as well as the larval integument's of Musca domestica vicina would be not only useful but also of prime importance. In addition to the effect of Melia azedarach on the larvae, pupae and adult of Musca vicina, the changes occurring in the cuticle of adult housefly after treatment with Melia azedarach extract had been also thoroughly studied (ElAhwany et al., 2016).
Numerous studies have been carried out on the insecticidal properties of different plant extracts. Members of the family Meliaceae represents one of the most important plants in this respect, as it contains the neem tree Azadirachta indica A. Juss and the chinaberry tree Melia azedarach (the common Egyptian name is Zanzalacht). The special properties of the toxic components of several Meliaceae species (including neem and chinaberry) and other plants used as repellents, antifeedants, and insect growth regulators, are of low cost (Radwan 1999, Ochi et al., 2020). Radwan (1999) found that the leaves and seeds of Melia azedarach display feeding inhibition for the house fly. The antifeeding and growth inhibiting effects of neem derivatives against a wide range of insects. Chinaberry also possesses such properties. A large number of structural variants have been isolated from Azadirachta indica, and other Meliaceae species like Melia azedarach. Radwan (1999), reported that, leaves of Melia azedarach contained an unidentified feeding-deterrent and growth retardant.

Eissa et al., (2020a) found that, the extracts from seeds of the neem tree Azadirachta indica and Melia azedarach (L.) contain several active principles of which azadirachtin is so far, the most potent, as a feeding deterrent and a growth regulator. This extract is characterized by being relatively safe and inexpensive. Radwan (1999), studied the antifeeding deterrent activity of leaf, seed and oil extracts of both Azadirachta indica and Melia azedarach. Azadirachtin was first isolated, identified and characterized from Azadirachta indica. Eissa et al., (2020b) found that it is noteworthy that azadirachtin has also been found in the related species Melia azedarach L. (Chinaberry or Persianlilac). Radwan (1999) found a sterilizing effect of azadirachtin on the mature females of the house fly. The treatment of these insects with neem seed extracts also causes growth inhibition, malformation, mortality. Azadirachtin has both antifeeding and toxic properties similar to those of the oils of neem, chinaberry and custard apple. Insect growth regulatory properties acting upon physiological processes have been attributed to various fractions of neem and chinaberry oil. 
Eissa et al., (2020a) claimed that the triterpenoid azadirachtins, strongly interfere with the neuroendocrine control of insect hormone titers. Various adverse effects on the growth and development of Spodoptera lituralis by azadirachtin, methanol extract of kernel and neem oil have also been reported by Eissa et al., (2020b). Those authors observed that azadirachtin application to the last instar of Spodoptera lituralis had a juvenilizing effect led to the formation of larval - pupal intermediates. They concluded that the growth disruption effect of azadirachtin on insects was nothing but its juvenilizing effect as a result of its action on the endocrine system. Radwan (1999), Ochi et al. (2020) reported that, topical applications of azadirachtin, a purified extract from seeds of the Indian neem tree $A$ indica A. juss, to larvae of the Japanese beetle, Popillia japonica Newman, completely disrupted subsequent normal development to the adult stage. Radwan et al., (2019 a, b, c, d), reported that, neem seed extract, containing a tetranortriterpenoid isolated from the seeds of the neem tree Azadirachta indica A. Juss, reduced oviposition of the rice leaffolder, Cnaphalocrocis medinalis.

\section{Materials and methods}

Raising a colony of Musca domestica vicina: Musca vicina, dissected and examined in this study, were all produced from a colony raised at the laboratory of the Zoology Department, Faculty of Science, Alexandria University. The female flies usually lay their eggs on the milk pads. The pads containing the eggs are usually transferred to glass- jars containing fresh milk pads to provide food for the newly hatched larvae. The jars are tightly covered with finely perforated tin lids. The jars are, then, kept in the adjusted constant temperature room. The hatched larvae are left to grow and molt in the jars till they reach the third instar. Prior to pupation, the larvae aggregate at the dried upper surface of the milk pad which by this time become blown up into spongy mass due to the continual tunneling of the larvae inside. At this spongy dried upper surface, the larvae usually pupate. The pupae are left in these jars in order to complete development. The emerging adults are then transferred to the breeding cages to maintain the stock.
Extraction method: Fresh leaves and ripe fruits were collected from the chinaberry trees, Melia azedarach, found in the garden of the Faculty of Science, Alexandria University ElShatby. Leaves and fruits were pulverized by means of a hummer mill. Extraction was conducted in a $250 \mathrm{ml}$ Soxhlet apparatus using methanol as a solvent. However, no separation of fat materials was done. The extraction period lasted a total of 20 hours over a period of four days until the chinaberry leaves and fruits (inside the Soxhlet) became colorless.

Fertility was determined as the number of eggs hatched compared with total number of eggs laid. Fecundity was determined as the mean number of eggs laid/female in each test (Abdel Baky, 1990).

Feeding experiments: In order to study the effect of Melia azedarach L. on the developmental rate of adult female house fly of $M$. vicina. Serial sets of experiments were set up. These include the treatment of adults at ages of $24 \mathrm{hrs}$., 48hrs., and $72 \mathrm{hrs}$.; with different concentrations of fruits and leaves extractions. The desired concentrations of Melia azedarach $\mathrm{L}$, extractions were $(1.8 \%, 2.4 \%$ and $3.6 \%), 10 \mathrm{ml}$. of each concentration was added to the adult's feeding medium.

The first set of experiments includes feeding adult flies at age 24 hrs., with different concentrations of fruits and leaves extraction. In the second set of experiments, feeding of adult 48 at age hrs., with Melia azedarach L. extractions. The third set of experiments, was carried out by exposing the adults at age $72 \mathrm{hrs}$., to different concentrations of fruit and leaves extractions. Each set of experiment comprised 8 groups of adults. Each group consisted of 100 individuals. Each group was replaced in a cage containing a Petri-dish containing a piece of cotton and $50 \mathrm{ml}$. of milk. Six groups of these adults were treated with different concentrations of fruits and leaves extractions $(1.8 \%, 2.4 \%$ and $3.6 \%)$, respectively, and kept under treatment until the first oviposition. Groups of untreated adults were acted as the control. The control groups were provided with the standard diet alone; Groups I and la were provided with standard diet to which a dose of $1.8 \%$ of fruits and leaves extractions have been added, respectively. Groups II and IIa contained the standard diet to which $2.4 \%$ of Melia 
azedarach L. extractions (fruits and leaves) were added, respectively, groups III and IIIa contained the standard diet to which $3.6 \%$ of fruits and leaves extractions were added, respectively. Triplicate tests of each group were performed. The results of these tests and data obtained together with the average means of these triplicate were tabulated. The time required for the completion of the first gonotrophic cycle and the number of egg laying were recorded. The hatchability rate of egg was also recorded.

In each Petri-dish, the number of exuviae. It is therefore that, the exuviae were looked for in different Petri-dishes every 24 hours during each experiment. The time of the onset of the successive ecdysis as well as the time required for the completion of the larvae were recorded. The mean duration of each instar was calculated statistically according to the following equation: $x=\sum \frac{f i x i}{n}$, Where: $x i=$ time (duration of each larval instars), $\mathrm{fi}=$ number of larvae reached ecdysis at $\mathrm{xi}, \mathrm{n}=$ total number of larvae.

In order to find out any lethal effect of Melia azedarach extractions might have on the larvae; all the dead larvae belonging to the different larval instars were counted and recorded. The mortality and survival percentages of the various larval instars till the onset of pupation were also calculated. The pupae resulting in each of the three sets of experiments were collected and placed in marked Petri-dishes. The Petri-dishes were placed in the breeding cages for the observation of the subsequent adult emergence. Normal, abnormal and intermediates among the larvae, pupae and adults were recorded and tabulated.

Topical application experiments: Adult females at age 24, 48 and 72 hours were divided into 4 groups. The control group contained individual which were treated topically with 0.36 $\mu \mathrm{g} / \mathrm{ml} / \mathrm{insect}$ of distilled water. Group 1 contained individuals that were treated topically with a single dose of $1.8 \mu \mathrm{g} / \mathrm{ml} /$ insect of fruit extractions. Group 2 contained individual that were treated topically with a single dose of $2.4 \mu \mathrm{g} / \mathrm{ml} /$ insect of fruit extraction. Group 3 contained individual that were treated topically with $3.6 \mu \mathrm{g} / \mathrm{ml} /$ insect of fruit extractions. The time required for the completion of the first gonotrophic cycle and the number of egg laying were recorded. The hatchability rate of eggs was recorded. Normal, abnormal and intermediates among the larvae, pupae and adults were recorded and tabulated.

Deterrent index: Deterrent indeces were calculated as represented by Lundgren (1975):

Deterrent index $=\frac{B-A}{A+B} \times 100$, Where, $\mathrm{A}$ and $\mathrm{B}$ are the number of eggs laid in the treated and the control groups, respectively.

Histological examination of the cuticle of larvae and adults of Musca domestica:

The cuticle of Musca domestica larvae and adults 24 hours old, 48 hours and 72 hours age were histologically investigated in comparison with those of the controls of the respective old. The tested Musca domestica larvae and adults were cut at the end of the thorax into two parts to insure fixation. Then they were fixed in Gillson (Pantin, 1964) for 48 hours ( 2 changes). The fixed materials were washed in $70 \%$ alcohol several times and dehydrated through the ascending alcoholic series, cleared in xylol and embedded in paraffin wax. Sections were cut at $6 \mu$ thickness, mounted on clean slides, dewaxed and dehydrated as usual. The staining was performed after; Mallory (1936) (Pantin, 1964). Mallory's stain is demonstrated in its perfect and constant differentiation of the epicuticle (Dennell, 1946). Treated larvae and adults were examined and photographed. Examination was carried out under the ordinary light microscope for the purpose of detecting any change that might have occurred in the structure of the cuticle.

Data were subjected to student's T-test, F-test and least significant difference (L.S.D.) test (Snedecor and Cochron, 1967).

\section{Results}

\subsection{Results of feeding experiments:}

Data obtained from the first set of experiments were in tables 1 and 2 . In the first set of experiments the percentages of the 1 st instar larvae successfully completed its development and reached the $2^{\text {nd }}$ larval instar were found to be $60 \%, 57.6 \%$ and $51.2 \%$ for groups I, II, III, respectively, as compared with $96.3 \%$ for the control group (Table 1) and percentages of $73.7 \%$, $68.1 \%$ and $53.4 \%$ for groups Ia, IIa and IIIa, 
respectively, as compared with $96.5 \%$ for the control group (Table 2). It should be noted that the percentages of the second instar larvae reaching the third instar were $50 \%, 38.4 \%$ \& $25.6 \%$ for groups I, II, III, respectively, as compared with $95.5 \%$ for the control group (Table 1), and a percentage of $65.8 \%, 50 \%$ and $41.8 \%$ for groups Ia, IIa and IIIa, respectively, as compared with $96.3 \%$ for the control group (Table 2).

In the present study, three groups of adult Musca vicina at age 48 hours were treated with three different concentrations of Melia azedarach fruits and leaves extractions. The three concentrations were $1.8 \%, 2.4 \%$ and $3.6 \%$. The data obtained have been represented in tables 3 and 4 . In the second set of experiments, tables 3 \& 4 demonstrate the retarding effect of the various concentrations of Melia azedarach extraction on the adult females Musca vicina. In the second set of experiments the percentages of the $1^{\text {st }}$ instar larvae reaching the second instar larvae reaching the standard time were found to be $55.5 \%, 50.7 \%$ and $40 \%$ for groups I, II \& III, respectively, as compared with $97.6 \%$ for the control group (Table 3) and percentages of $57.3 \%, 54.5 \% \& 42.3 \%$ for groups Ia, IIa and IIIa, respectively, as compared with $96.6 \%$ for the control group. (Table 4).

It is evident, therefore, that the time required for the completion of the third larval instar has been prolonged by $18.6,29.3 \& 50.3$ hours for groups I, II and III, respectively (Table 3 ) and 15. 3, 20 and 37 hours for groups Ia, IIa and IIIa, respectively (Table 4). Tables 5 and 6 demonstrate the retarding effect of the various concentrations of Melia azedarach extraction on the adult Musca vicina. The number and percentages of the $1^{\text {st }}$ instar larvae reaching the second instar larvae reaching the successive instar at the standard time have been recorded in tables 5 and 6 . The number of the molts from the first to the second instar was determined. In the third set of experiments the percentages of the 1 st instar larvae reaching the 2 nd instar larvae reaching the standard time were found to be $56.9 \%, 45.1 \%$ and $40.2 \%$ for groups I, II and III, respectively, as compared with $97.8 \%$ for the control group (Table 5); and percentages of $61.5 \%, 57.2 \% \& 51.6 \%$ for groups Ia, IIa and IIIa, respectively, as compared with $98.8 \%$ for the control group. (Table 6). The percentages of the second instar larvae reaching the third instar were $52.5 \%, 41.2 \%$ and $35.2 \%$ for groups I, II and III, respectively, as compared with $97.1 \%$ for the control group (Table 5), and percentages of $60 \%, 49.8 \% \& 44.9 \%$ for groups Ia, IIa \& IIIa, respectively, as compared with $97.4 \%$ for the control group (Table 6).

The exposure of the various ages of adult Musca vicina to the three tested concentrations of Melia azedarach extractions as shown in the previous sets of experiments gave rise to a number of noticeable abnormalities. The data obtained from these experiments, clearly demonstrate that the adults reared on media containing Melia azedarach extraction seem to show some morphological variations of larvae, pupae and adults when compared with control groups as follows: 1- Larval abnormalities: Small larvae, Pigmented larvae. 2) Pupal abnormalities: Larval -pupal intermediates, Constricted pupae, Pigmented pupae, Small pupae. 3) Adult abnormalities, Half emerged adults.

\subsection{Results of topical application experiments:}

The data obtained from these experiments have been represented in tables $(7-9)$. Table 7 shows that the time required for completing the first gonotrophic cycle in the female groups 1,2 and 3 at age 24 hours that had been exposed to the previously mentioned concentrations of Melia azedarach fruit extraction was $86,75 \& 68$ hours respectively, as compared with 96 hours for the control group (Table 7). Table (8), also shows that the time required for completing the first gonotrophic cycle of females at the age of 48 hours was decreased. It was $88,80 \& 70$ hours in groups 1, 2 and 3, respectively, as compared with 98 hours for the control group (Table 8). It may be seen also from table 9 , that the time required for completing the first gonotrophic cycle of females at age 72 hours was decreased. It was 90, 85 and 75 hours in groups 1, 2 and 3, respectively, as compared with 97 hours for control group (Table 9). Tables (7, 8 and 9) also show that the number and the percentages of the hatched eggs were decreased in treated groups, 1,2 and3. Such percentages were found to be $85.2 \%, 76 \%$ and 60 $\%$ for groups 1, 2 and3, respectively as compared 
with $96.4 \%$ for the control group (Table 7). Also, it reached a percentage of $84.2 \%, 72 \%$ and $58.3 \%$ in groups 1, 2 and 3, respectively as compared with $96.5 \%$ in the control group (Table 8).

It may be seen from table 9, that the number and percentages of hatched eggs in groups 1, 2 and 3, were decreased. Such percentages were $86.9 \%, 76.9 \%$ and $64.0 \%$, respectively, as compared with $98.5 \%$ for the control group (Table 9). It may be seen also from tables (7, 8 and9), that the production of abnormal small larvae had decreased with the increase of the concentration of Melia azedarach fruit extraction. Tables $(7,8$ and 9) also show that the rate of normal pupae produced from treated females had decreased by increase the concentration of Melia azedarach fruit extraction. Such percentages were $69.5 \%$, $50.5 \%$ and $32.0 \%$ were for groups $1,2 \& 3$, respectively, as compared with $96.4 \%$ for the control group (Table 7).

Also, it should be noted from table 8 that the percentages of normal pupae from treated female were $71 \%, 55.5 \%$ and $37.1 \%$ in groups 1 , 2 and 3, respectively, as compared with $96.5 \%$ in the control group (Table 8). Table 9, also shows that the rate of normal pupae produced from treated females had decreased by the increase of the concentration of Melia azedarach fruit extraction, such percentages were $73.3 \%, 60 \%$ and $56.2 \%$ for groups 1,2 and 3, respectively, as compared with $98.5 \%$ for the control group (Table 9). Tables (7, 8 and 9), show that the percentages of the half emerging adults that became incapable of leaving the cocoon were $4.3 \%, 6.3 \% \& 9.3 \%$ in groups 1,2 and 3 , respectively, (Table 7). A percentage of $5.0 \%$, $8.8 \%$ and $12.8 \%$ also were obtained from groups 1, 2 and 3, respectively, (Table 8). Table 9, also shows that the percentages of the half emerging adults in the treated groups were $1.6 \%, 3 \%$ and $1.2 \%$, respectively, on the other hand, the percentages of the fully emerged adults from normal pupae were decreased by the increase of the concentration of Melia azedarach fruit extraction.

Effect of feeding treatment on the fecundity and fertility of Musca vicina: Table $(1,2 \& 3)$ : shows the fecundity and fertility of adult house fly, resulted after their treatment with different concentrations of Melia azedarach fruit and leaf extracts. There is a great reduction in the number of eggs laid per female after treatment. The results obtained can be summarized as follows:

1- The number of eggs deposited by females, was reduced.

2- Adult fecundity was greatly affected as there were three sets of experiments: The first set of experiments used adult females Musca vicina treated with fruit and leaf extracts of Melia azedarach at age 24 hours (Tables $1 \& 2$ ). The second set of experiments used adult females Musca vicina treated with fruit and leaf extracts of Melia azedarach at age 48 hours (Tables $3 \& 4)$. The third set of experiments used adult females Musca vicina treated with fruit and leaf extracts Melia azedarach at age 72 hours (Tables 5\&6).

The first set of experiments: adult females Musca vicina at age 24 hours, by using fruit and leaves extract of Melia azedarach. The average number of eggs laid per female were 108, 94 and 32 eggs in group I, II and III, respectively, with deterrent index equal to $12.2,19$ and $62.4 \%$ in group I, II and III, respectively, compared with the average of 138 eggs in the control group. Whereas the average percentages of fertility, were $69,55.3$ and $49.1 \%$, in group I, II and III, respectively, compared with the average percentage of $98.5 \%$ in the control group. The average number of eggs laid per female were 112.3, 102.3 and 82.6 eggs in group I, II and III, respectively, with deterrent index equal to $10.8,15.4$ and $25.2 \%$ in group I, II and III, respectively, compared with the average of 139.3 eggs in the control group. Whereas the average percentages of fertility were $72.9,64.5$ and $52 \%$ in group I, II and III, respectively, compared with the average percentage of $98.5 \%$ in the control group.

The second set of experiments: adult females Musca vicina at age 48 hours. by using fruit and leaves extract of Melia azedarach. The average number of eggs laid per female were 105, 85.6 and 31.3 eggs in groups I, II and III, respectively, with deterrent index equal to $14.3,23.9$ and 63.7 $\%$ in group I, II and III, respectively, compared with the average of 140.3 in the control group. 
Whereas the average percentages of fertility were 68.7, 53.3 and $48.5 \%$ in group I, II and III, respectively, compared with the average of $99.3 \%$ in the control group. The average number of eggs laid per females were 120, 110 and 96.3 eggs in groups I, II, and III, respectively, with deterrent index equal to $7.7,12$ and $18.6 \%$ in groups I, II and III, respectively, compared with the average of 140 eggs in the control group. Whereas the average percentages of fertility were 81.2, 70 and $56.3 \%$ in groups I ,11 and III, respectively, compared with the average percentage of $99.1 \%$ in the control group.

The third set of experiments: adult females, Musca vicina at age 72 hours. using fruit and leaves' extract of Melia azedarach. The average number of eggs laid per female were 107.7, 100 and 96 eggs in groups I, II and III, respectively, with deterrent index equal to 13.9, 17.7 and 19.7 $\%$ in group I, II and III, respectively, compared with the average of 142.6 eggs in the control group. Whereas the average percentages of fertility were $85,77.6$ and $62.2 \%$ in group I, II and III, respectively, compared with the average percentage of $98.8 \%$ in the control group. The average number of eggs laid per female were $140.3,137.7$ and 132.3 eggs in groups I ,11 and III, respectively, with detergent index equal to 0.4 , 1.1 and $3.3 \%$ in groups I, II and III, respectively, compared with the average of 141 eggs in the control group. Whereas the average percentages of fertility were $92.6,88.9$ and $84.9 \%$ in groups I, II and III respectively, compared with the average percentage of $99 \%$ in the control group.

Concerning fertility, the results showed that adults' fertility was also adversely affected as a result of treatment. Statistical analysis showed that, fertility differed significantly in the treated and the control groups and within the treated groups, the difference was highly significant at $(\mathrm{P}<0.05)$. As regard to the reproductive capacity of the first generation ( $\mathrm{Fl})$, it was found that, fecundity was not affected as a result of treatment but the fertility of this generation was greatly affected, where, partial sterility was observed.

Effect of Melia azedarach fruit and leaf of methanolic extract on the cuticle of larval and adult house fly Musca domestica vicina: The structure of the cuticle of the normal adult house fly Musca vicina has been investigated herein. It is found to be composed of an outer epicuticle executable and an inner end cuticle, where the epicuticle was not thrown into micro tubercles. The epicuticle is loaded with black pigments, while the endocuticle was horizontally laminated (Fig. 1). After feeding treatment with fruit and leaf extracts of Melia azedarach; the histological structure of the cuticle appeared to be almost the same as described by (Kennaugh, 1972), in the normal specimens (Fig. 2). In Mallory preparations, it appeared to be composed of an outer, pigmented epicuticle, and an inner laminated endocuticle. It is suggested, herein, that, the application of fruit and leaves extract of Melia azedarach, in the experiments conducted on the cuticle of Musca vicina may have affected the forces that bind the chitin and the proteins of the end cuticle leading to their separation. The cuticle of the larval-pupal intermediates had a unique structure which might mean that the anterior half of the cuticle was in the larval form, while the posterior part was in the pupal form.

Morphology and color of the cuticle: It was noticed that the lateral sides of the posterior part of the abdomen appears buff in color. The second and the fourth segments are composed wholly of intersegmental membrane (Fig. 1). The present study shows that if the epidermis is carefully scraped off the cuticle, the dark color of the cuticle remains as it is. This indicates that the dark appearance of the cuticle in the intact adult is due to pigments which are not present in the epidermis, but located in the epicuticle.

Surface pattern: The surface pattern of the dorsal side of the cuticle of the adult house fly Musca vicina, when mounted flat in glycerol and examined in surface view under the high power of the compound microscope, appears as minute black dots.

Histology: Longitudinal sections taken in the third instar larva Musca vicina show a structural uniformity of the cuticle. In other words, it did not show differentiation into hard sclerites and soft intersegmental membranes. Thus, it appears highly folded as seen in (Fig. 3). These folds, however, allow greater degree of flexibility to the larval intersegment. In the adult house fly Musca vicina, the second and fourth segments are 
composed wholly of intersegmental membrane (Fig 1). In unstained longitudinal sections, the cuticle of the third larval instar and the adult house fly of Musca vicina showed only two layers, an inner laminated endocuticle possessing no pore canals and an outer epicuticle.

The epicuticle of the third instar larva and the epicuticle of the adult house fly Musca vicina is not thrown into micro tubercles. Regarding the histology of the larval and adult epicuticle of Musca vicina, it has been found to consist, as in most arthropods, of the four well known layers, they are, an innermost cuticulin layer, a very thin paraffin containing layer, a wax layer and an outermost cement layer. The cuticulin and paraffin layers of the epicuticle are similar to those described in other arthropods. The wax and cement layers are not distinctly separated from each other, and will be described as a single layer.

Paraffin sections of the larval cuticle of Musca vicina and the adult cuticle as well, often show a distinct layer separated or partly separated from the surface of the cuticle. Its granular appearance and the ease with which it is separated readily distinguishes it from the epicuticular layers, it is stained blue with Mallory's triple stain. The appearance of the cement layer, in the larval and adult epicuticle of Musca vicina, in paraffin sections, of material fixed in Gilson's mercury nitric mixture, shows a homogeneous sheet extending over the surface of the cuticle. It exhibited a finely granular appearance. Neither the gland vesicles nor their ducts were demonstrated in either the larval or adult cuticle of Musca vicina. It is therefore suggested that the cement and wax layers may be due to the secretions of special oenocytes.

The endocuticle: Unstained longitudinal sections of the third larval instar and the adult house fly Musca vicina showed the endocuticle as a colorless, non-refractile and horizontal laminated layer. It did not show any differentiation into outer and inner layer. Stained sections, however, showed the endocuticle stained blue with Mallory. In neither unstained nor stained preparations could the pore canals be detected in the endocuticle in both larvae and adult stages. Microscopic examination of the surface pattern of an abdominal cuticular sheet, from both sexes of
Musca vicina does not show the presence of similar areas.

The unstained longitudinal paraffin sections taken in the adult and larval abdominal sclerites of Musca vicina, showed only two principal layers namely, an outer amber colored layer and an inner colorless layer.

The outer executable is characterized not only by its homogeneity but also by its refractivity and brittleness. This brittleness often causes the breakdown of that layer into fragments during sectioning. The end cuticle, on the other hand, is characterized by being non retractile and horizontally laminated (Figs. 1\& 4).

The intersegment membrane has the same histological structure in both sexes. Histological, it is not basically different from that of the scleroses. It possesses an executable and end cuticle as being the principal layers. But, at the same time it should be mentioned here that, the anterior margin of the executable is broken up into wedge-shaped (Fig. 1) instead of being uniformly plane as it is the case in the sclerotic. Therefore, it will be seen that the end cuticle increases in thickness at the expense of the thickness of the executable. These structures disappear completely in the middle region of the intersegment membrane where the relative thickness of its cubicula layers gradually decreases. This variation in thickness allows the degree of flexibility needed to the intersegment membrane. Thus, the executable of the intersegment membrane, although not uniformly thick, is consistently thinner than the executable of the normal scleroses. In the specimen studied, herein, there is no arthropodal membrane condition. It should be noted that; the executable extends uninterrupted along the whole length of the intersegment membrane.

Staining reactions: The distinction between the two layers of the cuticle that has been observed in the unstained preparations had been strongly emphasized after staining with Mallory's triple stain. The executable was found to stain red while the end cuticle stained blue. This was observed in both scleroses and intersegment membranes.

The histology of the outer layer of the cuticle: The histological visible layers: The cuticulin layer, which forms the main bulk of the epicuticle, 
assumes an amber coloration like that of the underlying executable in both the scleroses and the intersegment membranes. It cannot be easily demonstrated with the ordinary histological methods, from the underlying executable. If the hard cuticle is placed in (Chlorine dioxide in $50 \%$ acetic acid) for several weeks, the amber coloration is bleached; and the cuticle becomes soft. The cuticulin layer shows a uniform affinity towards the acid fuchsine of Mallory's triple stain. The paraffin containing layer in both the sclerotic and the intersegment membrane cannot be distinguished easily as when it appears, it appears as a very thin line staining vivid red over the

Table 1: development of the larvae of Musca domestica vicina, produced from adult females fed on Melia azedarach fruit extract at age 24 hours, reaching the succeeding instar .

\begin{tabular}{||l|c|c|c|c|c|c||}
\hline \multicolumn{2}{|c|}{ Mean larval development produced by Musca domestica vicina females survived feeding on M. azedarach extract } \\
\hline \multirow{2}{*}{ Feeding medium provided } & \multicolumn{2}{|c|}{ First instar } & \multicolumn{2}{c||}{ Second instar } & \multicolumn{2}{c||}{ Third instar } \\
\cline { 2 - 7 } & \multicolumn{2}{|c|}{ After 72 hours } & \multicolumn{2}{c||}{ After 120 hours } & \multicolumn{2}{c||}{ After 168 hours } \\
\cline { 2 - 7 } & No. & $\%$ & No. & $\%$ & No. & $\%$ \\
\hline Standard diet & 135 & 99.2 & 133 & 96.3 & 130 & 95.5 \\
\hline Standard diet+1.8\% fruit extract. & 60 & $80.4^{*}$ & 45 & $60^{*}$ & 40 & $50^{*}$ \\
\hline Standard diet+2.4\% fruit extract. & 40 & $76.9^{*}$ & 30 & 57.6 & 20 & $38.4^{*}$ \\
\hline Standard diet+3.6\% fruit extract. & 11 & $70.5^{*}$ & 8 & $51.2^{*}$ & 42 & $5.6^{* *}$ \\
\hline
\end{tabular}


Table 2: $\quad$ Development of the larvae of Musca domestica vicina, produced from adult females fed on Melia azedarach leaf extract at age 24 hours, reaching the succeeding instar.

\begin{tabular}{|c|c|c|c|c|c|c|}
\hline \multicolumn{7}{|c|}{ Mean larval development produced by Musca domestica vicina females survived feeding on M. azedarach extract } \\
\hline \multirow{3}{*}{ Feeding medium provided } & \multicolumn{2}{|c|}{ First instar } & \multicolumn{2}{|c|}{ Second instar } & \multicolumn{2}{|c|}{ Third instar } \\
\hline & \multicolumn{2}{|c|}{ After 72 hours } & \multicolumn{2}{|c|}{ After 120 hours } & \multicolumn{2}{|c|}{ After 168 hours } \\
\hline & No. & $\%$ & No. & $\%$ & No. & $\%$ \\
\hline Standard diet & 135 & 98.5 & 132 & 96.3 & 132 & 96.3 \\
\hline Standard diet $1.8 \%$ leaf extract. & 70 & $85.3^{*}$ & 60 & $73.1^{*}$ & 54 & $65.8^{*}$ \\
\hline Standard diet $+2.4 \%$ leaf extract. & 53 & $80.3^{*}$ & 45 & $68.1^{*}$ & 33 & $50 *$ \\
\hline Standard diet $+3.6 \%$ leaf extract. & 33 & $76.7^{*}$ & 23 & $53.4 *$ & 18 & $41.8^{*}$ \\
\hline
\end{tabular}

Table 3: Development of the larvae of Musca domestica vicina, produced from adult females fed on Melia azedarach fruit extract at age 48 hours, reaching the succeeding instar.

\begin{tabular}{|l|c|c|c|c|c|c||}
\hline \multicolumn{3}{|c|}{ Mean larval development produced by Musca domestica vicina females survived feeding on M. azedarach extract } \\
\hline \multirow{2}{*}{ Feeding medium provided } & \multicolumn{2}{|c|}{ First instar } & \multicolumn{2}{c||}{ Second instar } & \multicolumn{2}{c||}{ Third instar } \\
\cline { 2 - 8 } & \multicolumn{2}{|c|}{ After 72 hours } & \multicolumn{2}{c||}{ After 120 hours } & \multicolumn{2}{c||}{ After 168 hours } \\
\cline { 2 - 8 } & No. & $\%$ & No. & $\%$ & No. & $\%$ \\
\hline Standard diet & 138 & 99 & 136 & 97.6 & 135 & 96.9 \\
\hline Standard diet+1.8\% fruit & 56 & $77.7^{*}$ & 40 & $55.5^{*}$ & 36 & $50^{*}$ \\
\hline extract. & 33 & $72.8^{*}$ & 23 & $50.7^{*}$ & 18 & $39.7^{*}$ \\
\hline $\begin{array}{l}\text { Standard diet+2.4\% fruit } \\
\text { extract. }\end{array}$ & 10 & $66.6^{*}$ & 6 & $40^{*}$ & 4 & $26.6^{*}$ \\
\hline $\begin{array}{l}\text { Standard diet+3.6\% fruit } \\
\text { extract. }\end{array}$ & & & & & \\
\hline
\end{tabular}


Table 4: $\quad$ Development of the larvae of Musca domestica vicina produced from adult females fed on Melia azedarach leaf extract at age 48 hours reaching the succeeding instar.

\begin{tabular}{||l|c|c|c|c|c|c||}
\hline \multicolumn{2}{||}{ Mean larval development produced by Musca domestica vicina females survived feeding on M. azedarach extract } \\
\hline \multirow{2}{*}{ Feeding medium provided } & \multicolumn{2}{|c|}{ First instar } & \multicolumn{2}{c||}{ Second instar } & \multicolumn{2}{c||}{ Third instar } \\
\cline { 2 - 8 } & \multicolumn{2}{|c|}{ After 72 hours } & \multicolumn{2}{c||}{ After 120 hours } & \multicolumn{2}{c||}{ After 168 hours } \\
\cline { 2 - 8 } & No. & $\%$ & No. & $\%$ & No. & $\%$ \\
\hline Standard diet & 136 & 98.1 & 134 & 96.6 & 134 & 96.6 \\
\hline Standard diet+1.8\%leaft \\
extract.
\end{tabular}

Table 5: $\quad$ Development of the larvae of Musca domestica vicina, produced from adult females fed on Melia azedarach fruit extract at age 72 hours, reaching the succeeding instar at standard time.

\begin{tabular}{||l|c|c|c|c|c|c||}
\hline \multirow{2}{*}{ Mean larval development produced by Musca domestica vicina females survived feeding on M. azedarach extract } \\
\hline \multirow{2}{*}{ Feeding medium provided } & \multicolumn{2}{|c|}{ First instar } & \multicolumn{2}{c||}{ Second instar } & \multicolumn{2}{c||}{ Third instar } \\
\cline { 2 - 8 } & \multicolumn{2}{|c|}{ After 72 hours } & \multicolumn{2}{c||}{ After 120 hours } & \multicolumn{2}{c||}{ After 168 hours } \\
\cline { 2 - 8 } & No. & $\%$ & No. & $\%$ & No. & $\%$ \\
\hline Standard diet & 139 & 98.5 & 138 & 97.8 & 137 & 97.1 \\
\hline $\begin{array}{l}\text { Standard diet+1.8\% fruit } \\
\text { extract. }\end{array}$ & 73 & $79.9^{*}$ & 52 & $56.9^{* *}$ & 48 & $52.5^{* *}$ \\
\hline $\begin{array}{l}\text { Standard diet+2.4\% fruit } \\
\text { extract. }\end{array}$ & 56 & $72.1^{*}$ & 35 & $45.1^{* *}$ & 32 & $41.2^{* *}$ \\
\hline $\begin{array}{l}\text { Standard diet+3.6\% fruit } \\
\text { extract. }\end{array}$ & 42 & $70.4^{* *}$ & 24 & $40.2^{* *}$ & 21 & $35.2^{* *}$ \\
\hline
\end{tabular}


Table 6: $\quad$ Development of the larvae of Musca domestica vicina, produced from adult females fed on Melia azedarach leaf extract at age 72 hours, reaching the succeeding instar.

\begin{tabular}{|c|c|c|c|c|c|c|}
\hline \multicolumn{7}{|c|}{ Mean larval development produced by Musca domestica vicina females survived feeding on $M$. azedarach extract } \\
\hline \multirow{3}{*}{ Feeding medium provided } & \multicolumn{2}{|c|}{ First instar } & \multicolumn{2}{|c|}{ Second instar } & \multicolumn{2}{|c|}{ Third instar } \\
\hline & \multicolumn{2}{|c|}{ After 72 hours } & \multicolumn{2}{|c|}{ After 120 hours } & \multicolumn{2}{|c|}{ After 168 hours } \\
\hline & No. & $\%$ & No. & $\%$ & No. & $\%$ \\
\hline Standard diet & 138 & 98.8 & 138 & 98.8 & 136 & 97.4 \\
\hline Standard diet $+1.8 \%$ leaf extract. & 106 & $81.5^{*}$ & 80 & $61.5^{*}$ & 78 & $60 *$ \\
\hline Standard diet $+2.4 \%$ leaf extract. & 97 & $79.3^{*}$ & 70 & $57.2^{*}$ & 61 & $49.8^{*}$ \\
\hline Standard diet $+3.6 \%$ leaf extract. & 85 & $75.6^{*}$ & 58 & $51.6^{*}$ & 55 & $44.9^{*}$ \\
\hline
\end{tabular}


Table 7: Gonotrophic cycle time, number of eggs laid, hatched and the development of the first generation produced from topically applied females of Musca domestica vicina age 24 hours treated with Melia azedarach fruit extract.

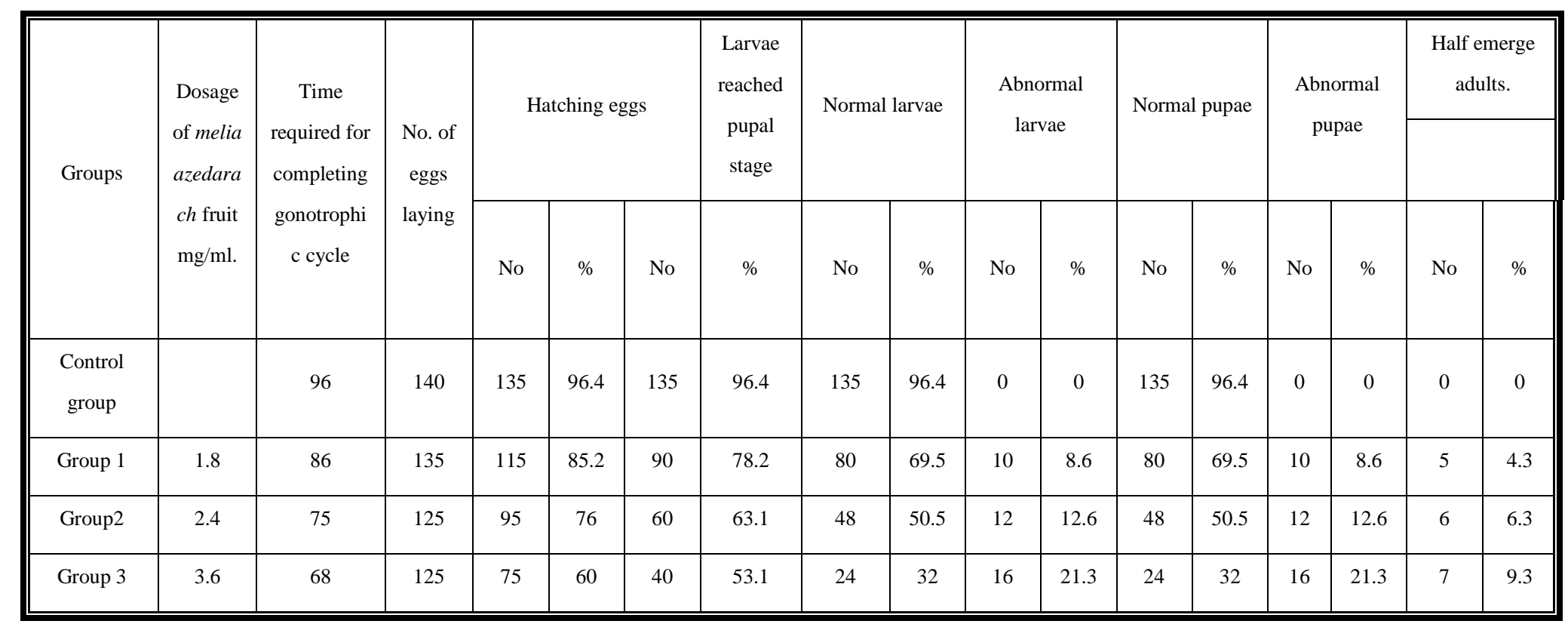

Table 8: Gonotrophic cycle time, number of eggs laid, hatched and the development of the first generation produced from topically applied females of Musca domestica vicina age 48 hours treated with Melia azedarach fruit extract

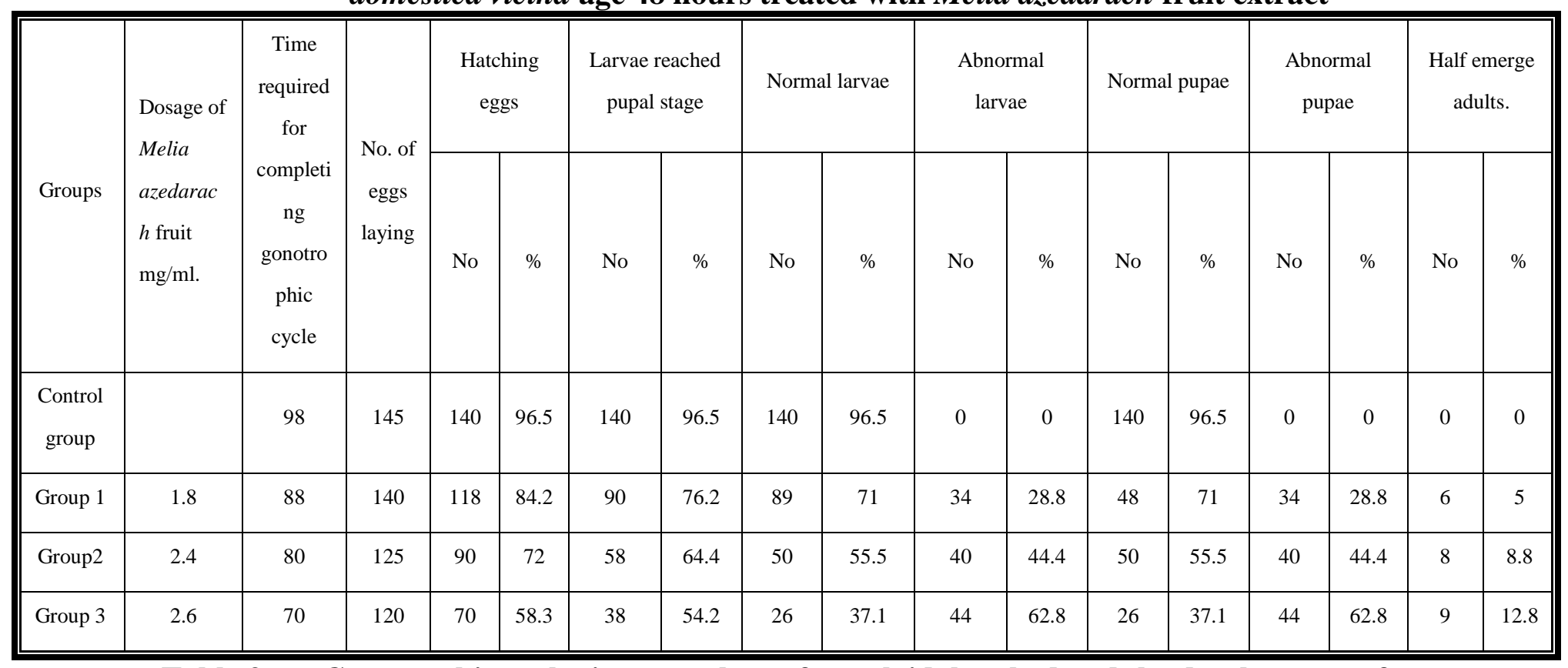

Table 9: Gonotrophic cycle time, number, of eggs laid, hatched and the development of the first generation of larvae and pupae producing from topically applied females of Musca domestica vicina at age 72 hours, treated with: 


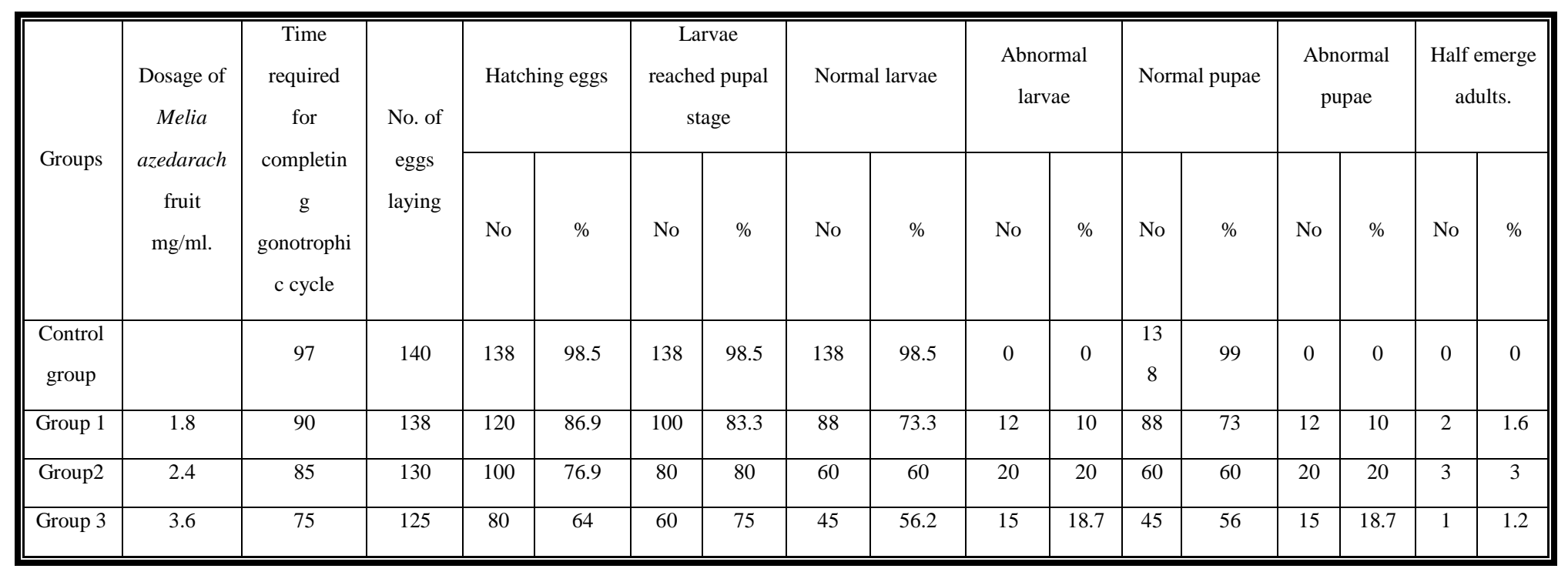

\section{Melia azedarach fruit extract}

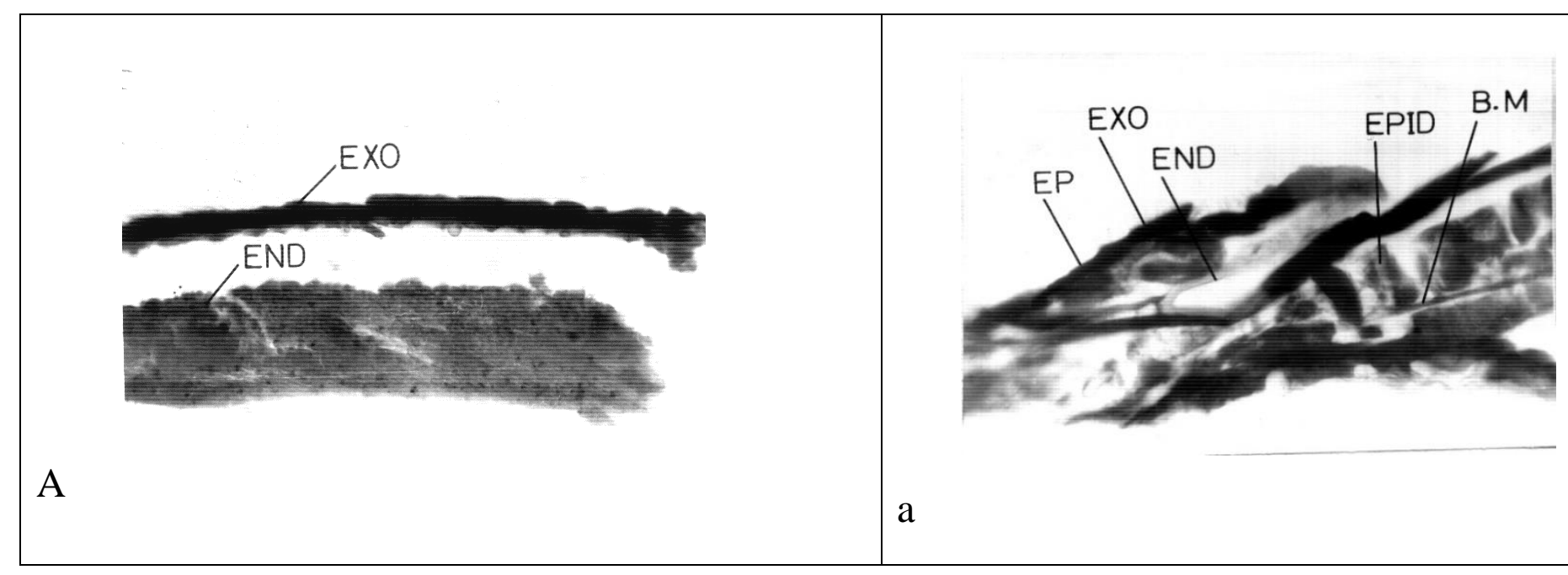




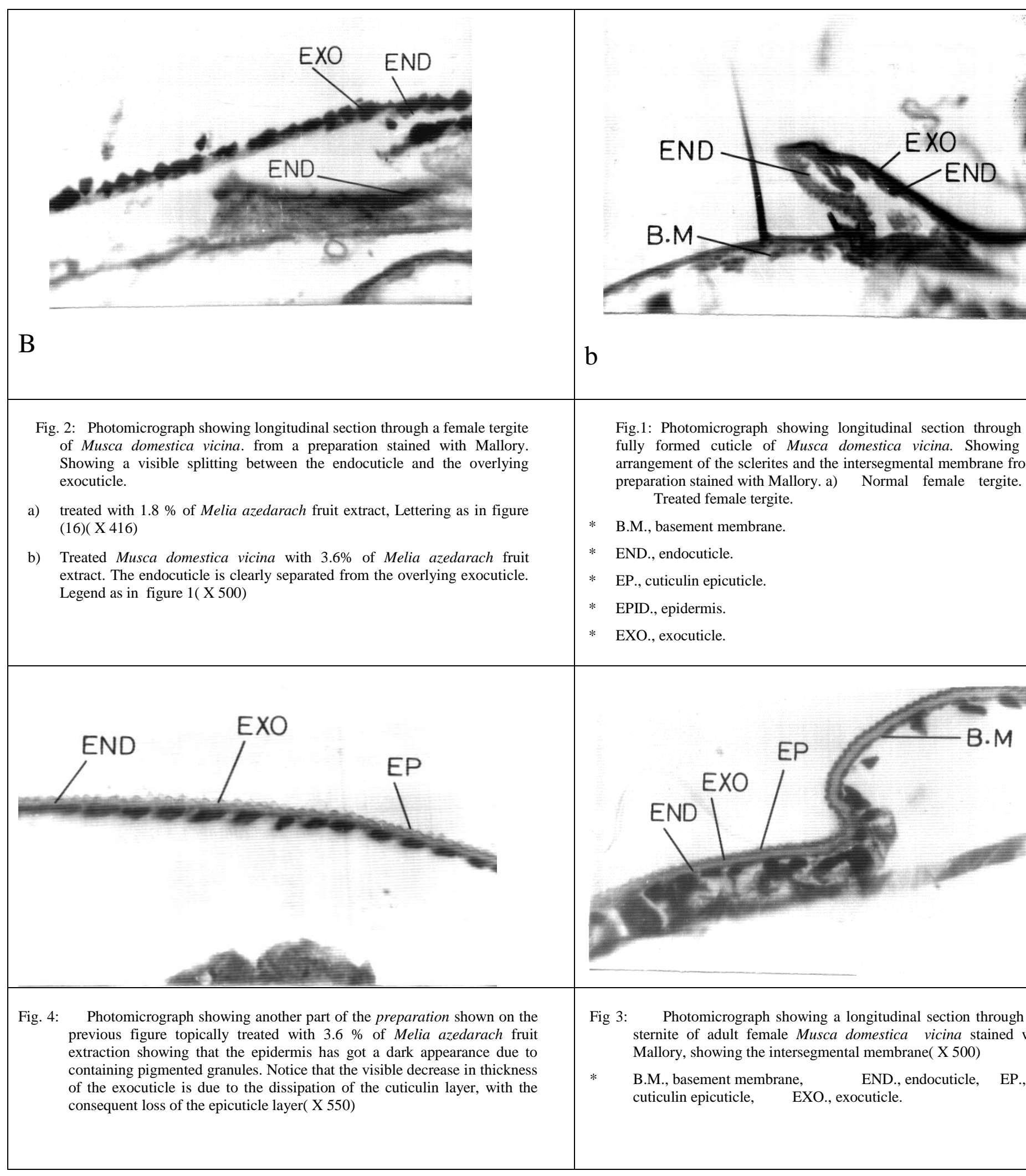




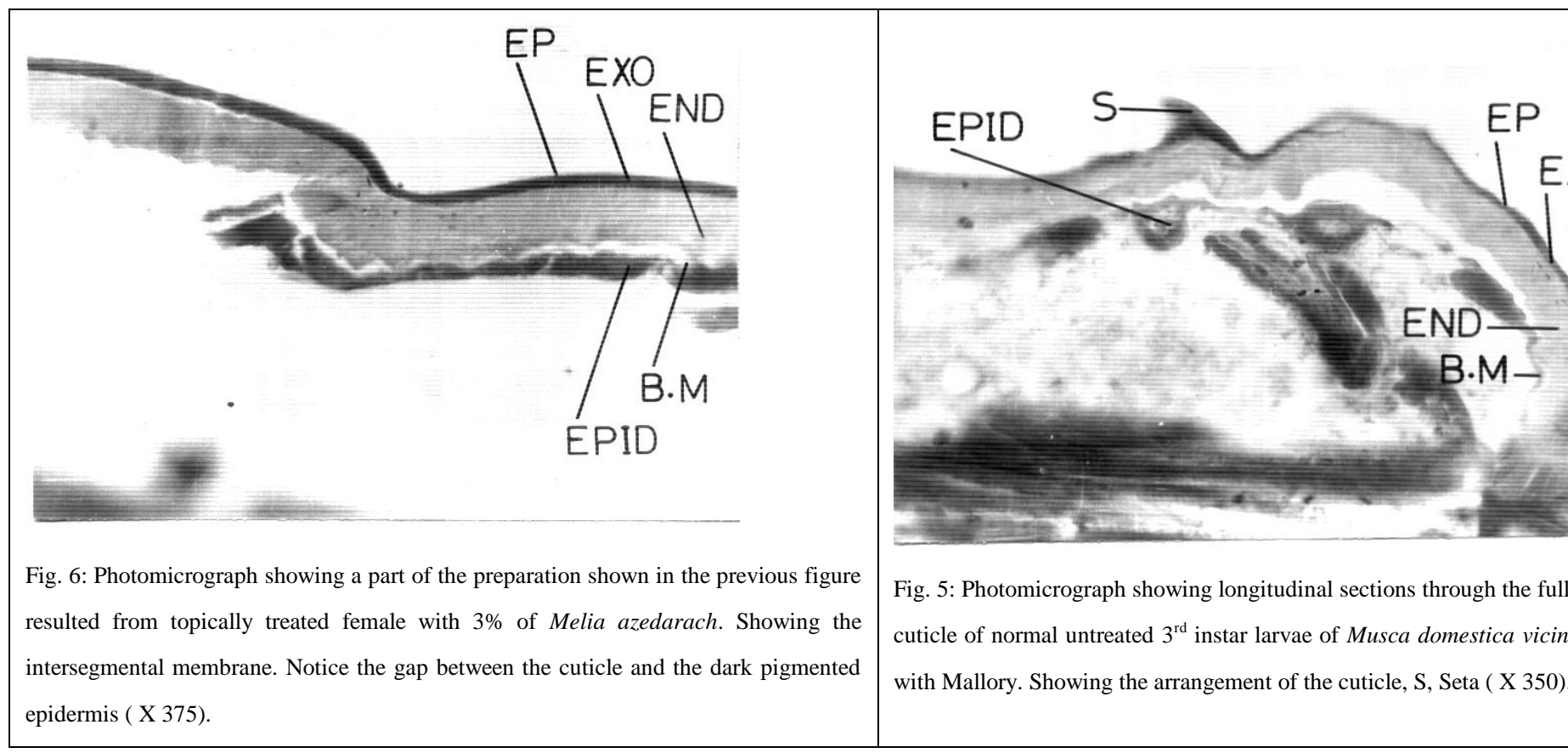

\section{Discussion}

Results obtained indicate the retarding effect of the fruits and leaves extracts of Melia azedarach, on female house fly Musca vicina at different ages 24, 48 and 72 hours, when they were treated with various concentrations of Melia azedarach extractions mainly $1.8 \% \quad 2.4 \%$ and $3.6 \%$. Eissa et al., 2020 found that azadirachtin significantly reduced fecundity and reproduction of Spodoptera litura. Radwan (2018) found that ethanolic extracts of neem seed reduced insect' oviposition. Rice and Coat (1994), treated adults of Musca domestica and their eggs with 22 monoterpenoids to determine the topical, fumigant.

Saxena et al. (1996), noticed that, topical treatment of Musca domestica L. with the phytochemical plumbagin (isolated from Plumbage zeylanic), in doses of 0.005 $5 \mathrm{Mg}$, prevented oocyte development and drastically affected fecundity and fertility in adults. Also, treatment of "Wandering" larvae was less effective, as the compound only affected fertility, not fecundity. It is clear from the data cited in tables (1-6) that oral administration of Melia azedarach to the adult house fly causes prolongation in the life span of the larval instar of the first generation. Eissa et al., 2020a, b, Radwan (2018) found that, application of Azadirachtin on various stages of and Spodoptera littura larvae caused larval mortality.

Hashem and Youssef (1991), studied the effect of methanolic extractions of leaves and fruits of Melia azedarach L. on the house fly Musca vicina Macq. They found that, the reaction of larval instars to the concentrations of Melia azedarach extractions is doses dependent. It was evident that the prolongation in the larval life span which occurred as a result of the exposure to Melia azedrarch extraction, encouraged researchers to make use of Melia azedarach extractions as larvicides.

Naqvi et al. (1990), Radwan (2018) studied the toxicity of the neem [Azadirachta indica] pesticide Margosan (containing $0.3 \%$ azadirachtin), the neem compounds NC (Nimolicin, an isolate O- 
neem fruits) and $\mathrm{H}-34$ (obtained by the alkaline hydrolysis of nimolin, triterpenoid of neem), were compared with the toxicity of a pyrethroid, Solfa (cyfluthrin) and two OP pesticides, DDVP (dichlorovos) and perfekthion (dimethoate) to adults of $\mathrm{Musca}$ domestica. They noticed that, the $\mathrm{OP}$ pesticides were the most toxic. Also, they showed that the order of efficacy was dichlorovous> dimethoate> cyfiuthrin > Margosano > Nimolicin> H-34. The pesticidal properties of juliflorine (derived from Prosopic julifora) and Margosano (derived from Azadirachta indica, containing azadirachtin as an active ingredient) were determined against $3^{\text {rd }}$ instar larvae of Musca domestica by Jahan et al. (1990).

Naqvi et al. (1994) found that $\mathrm{LD}_{20}$ of dektamethrin (25 WP) and a neem extract (Azadirachta inorphogenic) after 24 hours treatment was $1.56 \%$ and $13.5 \%$, respectively. Also, they noticed that, the adults which emerged successfully possessed various abnormalities. In many cases half-emerged adults and abnormal pupae were also observed. Naqvi et al. (1995), studied the toxicity of the pyrthroid Coopex 25 EC (permethrin) and a neem extract $\mathrm{N}-7$ against the $3^{\text {rd }}$ instar larvae of the house fly, Musca domestica. They found that the $\mathrm{LD}_{50}$ values of both compounds were $0.029 \%$ and $3.8 \%$, respectively, which revealed that pyrthroid was more toxic than N-7. Also, both compounds caused morphogenic effects on various stages of Musca domestica, including weight reduction and abnormal development. The first observed abnormality was the appearance of pigmented larvae.

It is indicated that neem products had some effect on proteins. The pesticidal properties of juliflorine (derived from Prosopic julifora) were administered against the $3^{\text {rd }}$ instar larvae of Musca domestica by Jahan et al. (1990). The teratogenic effects of these pesticides on larvae, pupae and adults were observed. Pereira and Guradutt (1990) used, petroleum ether extracts of [Clerodendrum inerme] leaves which afforded a compound that matched the compound (-)-3-epicaryoptin in physical spectral characteristics. Hashem and Youssef (1991) and Naqvi et al. (1994) studied the effect of in ethanolic extractions of leaves and fruits of Melia azedarach L. on the house fly Musca vicina Macquart. They noticed also that, the fruit extraction was more effective on the larvae than that of leaves. They also recorded that larvae, pupae and adults displayed morphological abnormalities as well as pronounced anomalies.

Also, Naqvi et al. (1995), studied the toxicity of the pyrethroid Coopex 5EC (Permethrin) and a neem extract N-7 against the $3^{\text {rd }}$ instar larvae of the house fly, Musca domestica. They found that the LD 50 values of both compounds were $0.029 \%$ and $3.8 \%$, respectively, which revealed that pyrethroid was more toxic than N-7. The abdominal cuticle of the adult house fly Musca vicina is not uniform in structure. It is differentiated into sclerites and flexible intersegmental membranes.

Only two principle layers of the adult house fly's cuticle Musca vicina were noticible. These layers are, an inner cuticulin layer, paraffin-containing layers, the wax layer and an outermost cement layer. Of these layers, only the cuticulin layer and paraffin-containing layer can he distinguished histologically in the specimens under investigation. The paraffin containing layer in both the sclerite and the intersegmental membrane cannot be distinguished easily it appears as a very thin line staining vivid red over the cuticulin layer after being stained with Mallory's triple stain.

The microscopic examination of the treated pieces of the abdominal adult cuticle 
were examined microscopically and found to be unequivocal in showing the pore canals. The fruit and leaves extract of Melia azedarach are effective against house flies Musca vicina (El Ahwany et al., 2016, Radwan 2019, a, b, c, d, Eissa et al., 2020a, b).

\section{Conclusion}

The metamorphosis was prolonged. The total larval stage had been prolonged by periods ranging from 8.3 to 50.3 hours depending on the concentration of Melia azedarach fruit and/or leaf extractions used and on the age of the treated adult female house fly Musca vicina. Various responses were exhibited by the different stages towards the different concentrations of Melia azedarach fruit and leaf extracts. A separation between the endocuticle and the overlying exocuticle was noticed. More extensive laboratory experiments are needed with the methanolic extracts of leaves and fruits of Melia azedarach on the abdominal cuticle of the house fly Musca vicina in order to detect the real damage in the cuticle thickness.

\section{References}

Abdel Baky, S.M. (1990). Biological behavior of the cigarette beetle, Lasioderma serricorne (F.), emerging from gamma irradiated larval or pupal stages Bull. Ent. Soc. Egypt, Econ. Ser. 18: 163-170.

Bartlett, A.C. (1990). Insect sterility, insect genetics, and insect control. CRC Handbook of pest management in agriculture. $2^{\text {nd }}$, V. (2) 279-287.

El Ahwany A.M.D., Radwan, E.H., Beltagy B.I. and Abo El Naga, O.A. (2016). Prevalence of bacterial isolates possibly transmitted by different areas in Damanhur city, E 1 Behara, Egypt and its impact on Musca domestica cuticle. Journal of Microbiology and Biological Sciences. Vol. 4(1) pp.001-017.

http://www.meritresearchjournals.org/ mbs/index.htm.

Eissa EE, EH Radwan, N Abdel Hakeem, KK Abdel Aziz, HO Hashem, KH Radwan (2020a). Impact if Chlorpyrifos on the Second Instar Mosquito Larvae as Bioindicator in El-Beheira Governorate, Egypt. International journal of limnology, Vol-1 Issue -1 Pg. no.- 1-13. DOI: 10.14302/issn.2691-3208.ijli-203268.

Eissa EE, S A Salama, K H Radwan, E H Radwan (2020b). Effect of Tetranortriterpenoid on the Fourth and Fifth Larval Instar of Spodoptera littoralis. J Bioinform Syst Biol 2020; 3 (1): 001-018. DOI: 10.26502/jbsb.5107010.

Hashem, H. O. and Youssef, N. S. (1991). Developmental changes induced by methanolic extracts of leaves and fruits of Melia azedarach L. on the house fly, Musca domestica vicina Macq. J. Egypt. Ger. Soc. Zool., 3: 335- 352.

Jahan, M., Ahmed, I. and Naqvi, S.N.H. (1990). Toxic and teratogenic effects of juliflorine and Margosan$\mathrm{O}$ on the Musca domestica L. larvae Department of ZoologyEntomology, University of Karachi, Pakistan. Proceedings of Pakistan Congress of Zoology. 1990, 10: 293-320.

Mallory, F.B. (1936). The aniline blue collagen Stain. Stain Technique. 11:101-102.

Naqvi, S.N.H., Tabassum, R., Jahan, M., Yasmen, N., Azmi, M.A. and Matin, Z., (1994). Toxicity and abnormalities produced by neem fraction (NM) and deltamelthrin against second instar larvae of Musca domestica L. (PCSIR 
Strain). Proceeding of PakistanCongress of Zoology. 1994, 14: 283-290; 31.

Naqvi, S.N.H., Jahan, M., Tabassum, R., Qamar, S.J. and Ahmed, I. (1995). Toxicity and teratogeny caused by Coopex $25 \mathrm{EC}$ and a neem extract $(\mathrm{n}-7)$ against $3^{\text {rd }}$ instar larvae of Musca domestica L. PAK. J. Zool., 27(1): 27-31.

Ochi, D.O., Umeuzuegbu, J.C., Mahmud, H., Ekebafe, L.O., Ani, MO (2020). Transesterification of neem (Azaderachta indica). Nigerian Research journal of chemical sciences, V (8), issue (1). Pp 91103.

Pantin, C.F.A. (1964). Notes on microscopical techniques for zoologists, Cambridge University Press.

Radwan (1999). Effect of methanolic extracts of leaves and fruits of chinaberry tree Melia azaderach L. on the adult house fly Musca domestica vicina Macq, MSc thesis. Alex. Univ. Egypt.

Radwan EH (2018). Chapter of Soil Toxicology: Potential Approach on the Egyptian Argo-Environment. Springer, DOI: 10.1007/698-2018242.

Radwan EH, HO Hashem, N S Youssef and Shalaby AM (2019b). The effects of Zanzalacht on the gonotrophic cycle of the adult house fly Musca domestica. Journal of plant and animal ecology. V (1), issue 2, pp 2339. DOI. 10.14302/issn.26376075.jpae-19-2586.

Radwan EH, A Hassan, Wael M Lotfy, A Abdel Mawgood and HM Mashaal (2019c). The prevalence of intestinal parasite infection in El Behara School children. International journal of Limnology. V (1), issue (1), pp. 3351.

Radwan EH, HO Hashem, N S Youssef and Shalaby AM (2019d). The effects of Zanzalacht on the gonotrophic cycle of the adult house fly Musca domestica. Journal of plant and animal ecology. V (1), issue 2, pp 2339 DOI. 10.14302/issn.26376075.jpae-19-2586.

Rice, P.J., Coats, J.R. (1994). Insecticidal properties of several monoterpenoids to the house fly corn root worm (Coleoptera: Tenebrionidae and southern corn root worm (coleoptera: chrysomelidae). J. of Econ. Entomol. 1994, 87:5, 1172-1179;23.

Roush, R.T. and Tabashnik, B.E. \{eds.\} (1990). Pesticide resistance in arthropods. Chapman and Hall New York.

Saxena, BY., Thappa, R.K., Tikku, K.., Sharma, A., and Sari, O.P. (1996). Effect of plumbagin on gonadotrophic cycle of the house fly, Musca domestica vicina L. India, J. of Exp. Biol. 1996, 34: 8, 739-744. 